\title{
ACOUSTIC IMAGING AND SUBSURFACE ABSORPTION STRUCTUR OF SUNSPOTS
}

\author{
H.-K. CHANG AND D.-Y. CHOU \\ Department of Physics, National Tsing Hua University, \\ Hsinchu 30043, Taiwan, ROC
}

B. LABONTE

Institute for Astronomy, University of Hawaii

Honolulu, HI 96822, USA

AND

THE TON TEAM

Recently, Chang et al. (1997, hereafter Paper I) developed a new method, based on time-distance curves (Duvall et al. 1993), to construct a three-dimensional acoustic intensity image of the solar interior. Here we report results from another data set.

Solar p-mode waves, which are continually generated and dissipated stochastically by the turbulent convection, are scattered and absorbed by local inhomogeneities. If we appropriately add p-mode waves observed at the surface, based on the knowledge of how the waves propagate (time-distance curves), such that acoustic signals emanating from a particular point at a particular time are collected in phase, we can reconstruct the p-mode amplitude at the target point at the target time. The scheme of adding signals from a target point in phase plays the same role as a lens in optics. Thus we call it the "computational acoustic lens" (Paper I). This imaging technique can be used to construct acoustic amplitudes at any point in the solar interior based on the time-distance curve between that depth and the surface, which can be computed from a standard solar model based on the ray theory. The time series of constructed amplitudes provides information of both the intensity and the phase of the wavetrain. The intensity can be used to study p-mode absorption regions, and the phase can be used to probe other properties of local inhomogeneities, such as flow and change in wave speed.

The helioseismic data in this study were taken by the Taiwan Oscillation Network (TON) in the period of July 29 - August 6, 1996. NOAA 7981 is selected as a target region. Detailed data reduction procedure is described in Chou et al. (1997). The acoustic signals constructed with a normal time-distance curve are those propagating outward from a target point since the normal time-distance curve describes outgoing waves. With a time-reversed time-distance curve, we can also construct incident acoustic intensity at a target point. The acoustic images constructed with a time-reversed time-distance curve have the same acoustic intensity as those constructed with a normal time-distance curve and show no signature of the sunspot as expected because 
incident waves in the surrounding area have not been affected by local properties of the target point before they reach it.

To study $\mathrm{p}$-mode absorption in active regions, we subtract the incident acoustic intensity from the outgoing intensity and then divide it by the incident acoustic intensity for each target point to obtain fractions of power of incident waves absorbed in the medium along wave paths. At the surface, the strongest absorption, $29.8 \%$, occurs in the preceding sunspot. We construct absorption images at various depths from the surface to $80 \mathrm{Mm}$ deep at an interval of $10 \mathrm{Mm}$. A collecting area of the same annular region, $2-14^{\circ}$, is used for all depths. These subsurface absorption images are qualitatively similar to the intensity images constructed with outgoing waves for NOAA 7973 (Paper I). The absorption feature in the sunspot disappears at about 50 $\mathrm{Mm}$ below the surface for NOAA 7981 and about $40 \mathrm{Mm}$ below the surface for NOAA 7973. Since the major component of the spatial fluctuation in the quiet Sun is believed to be noise, we can estimate the vertical spatial resolution from the correlation of the quiet Sun feature among different depths. The vertical spatial resolution for both regions is about $20 \mathrm{Mm}$. Thus we estimate that the depth of the absorption region for sunspots is about $20-30 \mathrm{Mm}$.

In addition to acoustic absorption images, the time series of constructed amplitudes can also provide phase information. The relative phase between two time serieses can be determined from the cross-correlation technique. Here mentioned are two results from the phase study. First, the phase of p-modes advances about one minute for each reflection at the surface. Second, the phase shift between the time serieses constructed by incident waves and those constructed by outgoing waves in the sunspot area is different from the quiet Sun. Phase information has a better vertical spatial resolution than intensity, and may soon prove to be a more powerful tool for probing solar subsurface structures.

HKC, DYC, and the TON project were supported by NSC of ROC under grants NSC-87-2112-M-007-044. BL was supported by NASA grant NAGW-1542 and NASA contract NAS8-37334 of USA.

\section{References}

Chang, H.-K., Chou, D.-Y., LaBonte, B., and the TON team: 1997, Nature 389, 825 (Paper I).

Chou, D.-Y., Chang, H.-K., Sun, M.-T., LaBonte, B., Chen, H.-R., Yeh, S.-J., and the TON team: 1997, $A p J$, submitted.

Duvall, T. L. Jr., Jefferies, S. M., Harvey, J. W., and Pomerantz, M. A.: 1993, Nature 362, 430 . 